\title{
SCRAWNY CANTOR SETS ARE NOT DEFINABLE BY TORI
}

\author{
AMY BABICH \\ (Communicated by James E. West)
}

\begin{abstract}
We define a Cantor set $C$ in $\mathbf{R}^{3}$ to be scrawny if for each $p \in C$ and each $\varepsilon>0$ there is a $\delta>0$ such that for each map $f: S^{1} \rightarrow \operatorname{Int} B(p, \delta)-$ $C$ there is a map $F: D^{2} \rightarrow \operatorname{Int} B(p, \varepsilon)$ such that $F \mid \partial D^{2}=f$ and $F^{-1}(C)$ is finite. We show the existence and explore some of the properties of wild scrawny Cantor sets in $\mathbf{R}^{3}$. We prove, among other things, that wild scrawny Cantor sets in $\mathbf{R}^{3}$ are not definable by solid tori.
\end{abstract}

\section{INTRODUCTION: HISTORICAL BACKGROUND}

This paper concerns the existence and properties of a class of wild Cantor sets in $\mathbf{R}^{3}$.

Any perfect, uncountable, zero-dimensional compact metric space is called a Cantor set, and any two Cantor sets are topologically equivalent. Since the publication of Antoine's Necklace in 1921 [A], it has been known that there are inequivalent embeddings of Cantor sets in $\mathbf{R}^{3}$; that is, there are Cantor sets $C_{1}$ and $C_{2}$ in $\mathbf{R}^{3}$ such that, for any homeomorphism $h: \mathbf{R}^{3} \rightarrow \mathbf{R}^{3} h\left(C_{1}\right) \neq h\left(C_{2}\right)$.

Definition. Let $C$ be a Cantor set in $\mathbf{R}^{3}$. If there is a homeomorphism $h: \mathbf{R}^{3} \rightarrow$ $\mathbf{R}^{3}$ such that $h(C)$ lies on a straight line then $C$ is called tame. Otherwise, $C$ is wild.

Definition. Let $C$ be a Cantor set in $\mathbf{R}^{3}$. Let $\left\{\mathscr{M}_{n} \mid n \in \mathbf{N}\right\}$ be a sequence of finite collections $\mathscr{M}_{n}=\left\{M_{n, k} \mid 1 \leq k \leq m(n)\right\}$ of disjoint connected PL 3-manifolds-with-boundary $M_{n, k} \subseteq \mathbf{R}^{3}$ such that

(1) for each positive integer $\bigcup \mathscr{M}_{n+1} \subseteq \operatorname{Int} \bigcup \mathscr{M}_{n}$;

(2) $\bigcap\left\{\bigcup \mathscr{M}_{n} \mid n \in \mathbf{N}\right\}=C$.

Then $\left\{\mathscr{M}_{n}\right\}$ is called a defining sequence for $C$.

The following result is well known.

Received by the editors May 30, 1990.

1991 Mathematics Subject Classification. Primary 57M30, 57M35.

The contents of this paper were presented at the Fifth International Topology Conference in Dubrovnik, Yugoslavia, in June, 1990, sponsored by the Union of Mathematicians, Physicists, and Astronomers of Yugoslavia. A travel grant from the National Science Foundation and the Association for Women in Mathematics enabled the author to attend the conference. 
Theorem A (Bing [Bi]). Let $C$ be a Cantor set in $\mathbf{R}^{3}$. Suppose that there is a defining sequence $\left\{\mathscr{M}_{n} \mid n \in \mathbf{N}\right\}$ for $C$ in $\mathbf{R}^{3}$ such that, for each positive integer $n$ and for each integer $1 \leq k \leq m(n), M_{n, k}$ is a 3-ball. Then $C$ is tame.

Notation. Let $x \subseteq \mathbf{R}^{3}$ and let $\varepsilon>0$. Then $B(x, \varepsilon)$ denotes the round 3-ball in $\mathbf{R}^{3}$ with center at $x$ and radius $\varepsilon$.

Notation. Let $X \subseteq \mathbf{R}^{3}$ and let $\varepsilon>0$. Then $N(X, \varepsilon)$ denotes the closure of the $\varepsilon$-neighborhood of $X$ in $\mathbf{R}^{3}$.

Another well-known tameness theorem is

Theorem B (Bing [Bi], Homma [H]). Let $C$ be a Cantor set in $\mathbf{R}^{3}$. Suppose that for each point $p$ of $C$ and each $\varepsilon>0$, there is $a \delta>0$ such that, given any map $f: S^{1} \rightarrow \operatorname{Int} B(p, \delta)-C$, there is a map $F: D^{2} \rightarrow \operatorname{Int} B(p, \varepsilon)-C$ such that $F \mid \partial D^{2}=f$. Then $C$ is tame.

A recent improvement on Theorem $B$ is

Theorem $\mathbf{C}$ (Babich [Ba]). Let $C$ be a Cantor set in $\mathbf{R}^{3}$. Suppose that for each point $p$ of $C$ and each $\varepsilon>0$, there are a positive integer $n$ and $a \delta>0$ such that, for each map $f: S^{1} \rightarrow \operatorname{Int} B(p, \delta)-C$, there is a map $F: D^{2} \rightarrow \operatorname{Int} B(p, \varepsilon)$ such that $F \mid \partial D^{2}=f$ and $F^{-1}(C)$ consists of $n$ points or fewer. Then $C$ is tame.

\section{Definition AND STATEMENTS OF MAIN THEOREMS}

Weakening the hypothesis of Theorem $\mathrm{C}$ leads to the following

Definition. Let $C$ be a Cantor set in $\mathbf{R}^{3}$. Suppose that for each point $p$ of $C$ and each $\varepsilon>0$, there is a $\delta>0$ such that, for each map $f: S^{1} \rightarrow \operatorname{Int} B(p, \delta)-$ $C$, there is a map $F: D^{2} \rightarrow \operatorname{Int} B(p, \varepsilon)$ such that $F \mid \partial D^{2}=f$ and $F^{-1}(C)$ is finite. Then $C$ is scrawny.

In this paper we shall prove the following two theorems.

Theorem 1. Let $C$ be a wild scrawny Cantor set in $\mathbf{R}^{3}$. Then for some point $x$ of $C$ and some $\varepsilon>0$, there is a nonsingular disk $D \subseteq \operatorname{Int} B(x, \varepsilon)$ such that

(a) $D \cap C=\operatorname{Int} D \cap C=\{x\}$,

(b) $[\partial D] \neq 1$ in $\pi_{1}(\operatorname{Int} B(x, \varepsilon)-C)$.

Theorem 2. Let $C$ be a wild scrawny Cantor set in $\mathbf{R}^{3}$. There is no defining sequence $\left\{\mathscr{M}_{n} \mid n \in \mathbf{N}\right\}$ for $C$ such that, for each positive integer $n$ and for each integer $1 \leq k \leq m(n), M_{n, k}$ is a solid torus.

\section{EXAMPLE OF A WILD SCRAWNY CANTOR SET}

Before we prove theorems about wild scrawny Cantor sets in $\mathbf{R}^{3}$, it behooves us to show that such Cantor sets exist.

Let $C$ be a Cantor set in $\mathbf{R}^{3}$ with defining sequence $\left\{\mathscr{M}_{n}\right\}$ such that, for each positive integer $n$ and each $1 \leq k \leq m(n)$,

(1) $M_{n, k}$ is a solid double torus (thickened figure 8),

(2) $M_{n, k} \cap \bigcup \mathscr{M}_{n+1}$ is as shown in Figure 1. 


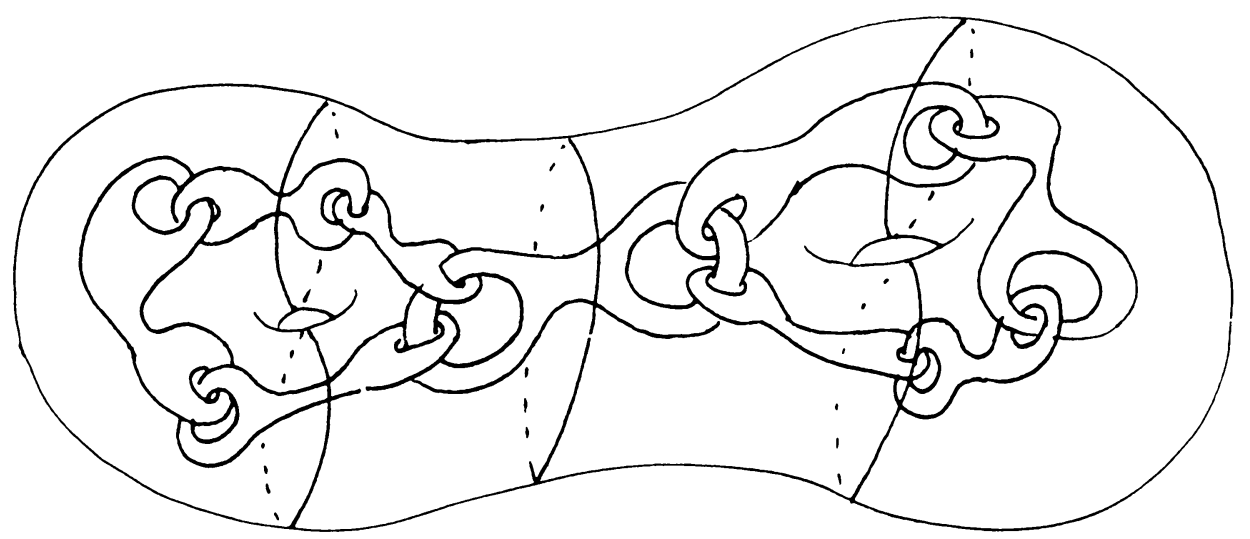

FIGURE 1

The Cantor set $C$ (which we will call Antoine's Eyeglasses) is a variation on Antoine's Necklace. That $C$ is wild can be shown by any of the many wellknown proofs of the wildness of Antoine's Necklace. (See, for instance, [B1], [D], or [M].) It is evident from the construction that Antoine's Eyeglasses is scrawny.

Thus, wild scrawny Cantor sets exist in $\mathbf{R}^{3}$. Not all wild Cantor sets in $\mathbf{R}^{3}$ are scrawny. Antoine's Necklace, for example, is not scrawny. Wild scrawny Cantor sets in $\mathbf{R}^{\mathbf{3}}$, then, are a nonempty proper subclass of wild Cantor sets in $\mathbf{R}^{3}$. Let us see what can be proved about them.

\section{MAIN TOOLS}

In the proofs that follow the main tools are the Loop and Sphere Theorems of Papakyriokopoulos and the following useful improvement on the Loop Theorem.

Plane Theorem (Brown and Feustel [BF]). Let $M$ be a noncompact 3-manifoldwith-boundary, and let $f: \mathbf{R}^{2} \rightarrow$ Int $M$ be a proper PL map. For each $n \in N$, let $S_{n}=\left\{x \in \mathbf{R}^{2} \mid\|x\|=n\right\}$. Let $K$ be a compact subset of $M$, and let $H$ be a normal subgroup of $\pi_{1}(M-K)$. Suppose that, for any large enough positive integer $n, f\left(S_{n}\right) \cap K=\varnothing$ and $\left[f \mid S_{n}\right] \notin H$.

Then there is a proper PL embedding $g: \mathbf{R}^{2} \rightarrow$ Int $M$ such that, for any large enough positive integer $n, g\left(S_{n}\right) \cap K=\varnothing$ and $\left[g \mid S_{n}\right] \notin H$.

We shall also use the following easy consequence of Theorem $\mathrm{A}$.

Theorem $\mathbf{D}$ (Bing [Bi]). Let $C$ be a wild Cantor set in $\mathbf{R}^{3}$. Then there are $p \in C$ and $\varepsilon>0$ such that, for each PL 3-ball $B \subseteq \operatorname{Int} B(p, \varepsilon), \partial B \cap C \neq \varnothing$.

\section{Proofs of MAIN THEOREMS}

Theorem 1. Let $C$ be a wild scrawny Cantor set in $\mathbf{R}^{3}$. Then for some point $x$ of $C$ and some $\varepsilon>0$, there is a nonsingular disk $D$ in $\operatorname{Int} B(x, \varepsilon)$ such that

(1) $\partial D \cap C=\varnothing$;

(2) $D \cap C=\{x\}$;

(3) $[\partial D] \neq 1$ in $\pi_{1}(\operatorname{Int} B(x, \varepsilon)-C)$. 
Proof. Since $C$ is wild, by Theorem $\mathrm{D}$ there is a point $p$ of $C$ and a $\theta>0$ such that, for any PL 3-ball $B \subseteq \operatorname{Int} B(p, \theta)$ such that $p \in \operatorname{Int} B, \partial B \cap C \neq \varnothing$.

Let $B$ be a PL 3-ball in $\operatorname{Int} B(p, \theta)$ such that $p \in \operatorname{Int} B$. Let $\eta>0$ such that $\eta<\operatorname{dist}(\partial B, p)$ and $N(\partial B, \eta) \subseteq \operatorname{Int} B(p, \theta)$. For each $\delta>0$, there is a PL simple closed curve $\alpha$ on $\partial B-C$ such that diam $\alpha<\delta$ and $[\alpha] \neq 1$ in $\pi_{1}(\operatorname{Int} N(\partial B, \eta)-C)$. For suppose, on the contrary, that there is a $\delta>0$ such that, for each PL simple closed curve $a$ on $\partial B-C$ such that diam $\alpha<\delta$, $[\alpha]=1$ in $\pi_{1}(\operatorname{Int} N(\partial B, \eta)-C)$. Since $C \cap B$ is compact and zero-dimensional, there is a finite collection $\left\{D_{i} \mid 1 \leq i \leq n\right\}$ of disjoint PL disks $D_{i}$ such that for each $1 \leq i \leq n$,
(a) $\partial D_{i} \subseteq \partial B-C$;
(b) $\operatorname{diam} D_{i}<\delta$;
(c) $\partial B \cap C \subseteq \bigcup\left\{\right.$ Int $\left.D_{i} \mid 1 \leq i \leq n\right\}$;
(d) $\left[\partial D_{i}\right]=1$ in $\pi_{1}(\operatorname{Int} N(\partial B, \eta)-C)$.

Condition (d) implies that for each $1 \leq i \leq n$, there is a PL map $f_{i}: D^{2} \rightarrow$ Int $N(\partial B, \eta)-C$ such that $f_{i} \mid \partial D^{2}=\partial D_{i}$ and $f_{i}^{-1}(C)=\varnothing$. Consider $\Sigma=$ $\partial B-\bigcup\left\{\operatorname{Int} D_{i} \mid 1 \leq i \leq n\right\} \cup \bigcup\left\{\operatorname{Im} f_{i} \mid 1 \leq i \leq n\right\}$. There is a PL map $\varphi: S^{2} \rightarrow$ Int $N(\partial B, \eta)-C$ such that $\operatorname{Im} \varphi=\Sigma$ and $[\varphi] \neq 0$ in $\pi_{2}(\operatorname{Int} B(p, \theta)-\{p\})$. By the Sphere Theorem of Papakyriokopoulos, there is a nonsingular PL 2-sphere $S$ in Int $N(\partial B, \eta)-C \subseteq \operatorname{Int} B(p, \theta)-C$ such that $[S] \neq 0$ in $\pi_{2}(\operatorname{Int} B(p, \theta)-$ $\{p\})$. By our choices of $p$ and $\theta$, this is impossible.

So for each $\delta>0$ there is a PL simple closed curve $\alpha$ on $\partial B-C$ such that $\operatorname{diam} \alpha<\delta$ and $[\alpha] \neq 1$ in $\pi_{1}(\operatorname{Int} N(\partial B, \eta)-C)$. Let $\left(\alpha_{k}\right)$ be an infinite sequence of disjoint PL simple closed curves on $\partial B-C$ such that for each positive integer $k$,

(1) $\left[\alpha_{k}\right] \neq 1$ in $\pi_{1}(\operatorname{Int} N(\partial B, \eta)-C)$,

(2) $\operatorname{diam} \alpha_{k}<1 / k$.

Since $\partial B$ is compact, the set $\bigcup\left\{\alpha_{k} \mid k \in \mathbf{N}\right\}$ has at least one limit point $y$ on $\partial B$. Since, for each positive integer $k, \operatorname{dist}\left(\alpha_{k}, C\right)<1 / k, y \in C$.

So there is a point $y$ of $\partial B \cap C$ such that, for each positive integer $k$, there is a PL simple closed curve $\alpha_{k}$ on $\partial B-C$ such that

(a) $\operatorname{dist}\left(\alpha_{k}, y\right)<1 / k$,

(b) $\left[\alpha_{k}\right] \neq 1$ in $\pi_{1}(\operatorname{Int} N(\partial B, \eta)-C)$.

The Cantor set $C$ is scrawny. So there is a $\delta>0$ such that, for each map $f: S^{1} \rightarrow \operatorname{Int} B(y, \delta)-C$, there is a map $F: D^{2} \rightarrow \operatorname{Int} B(y, \eta)$ such that

(1) $F \mid \partial D^{2}=f$,

(2) $F^{-1}(C)$ is finite.

For some positive integer $k, \alpha_{k} \subseteq \operatorname{Int} B(y, \delta)-C$. So there is a map $F: D^{2} \rightarrow \operatorname{Int} B(y, \eta)$ such that $F \mid \partial D^{2}=\alpha_{k}$ and $F^{-1}(C)$ consists of finitely many points.

We consider the finite set $F^{-1}(C) \subseteq \operatorname{Int} D^{2}$. There is a point $z$ of $F^{-1}(C)$ and a subdisk $D_{z}$ of $D^{2}$ such that

(a) $F^{-1}(C) \cap D_{z}=\{z\}$;

(b) $z \in \operatorname{Int} D_{z}$ and $D_{z} \subseteq \operatorname{Int} D^{2}$;

(c) $\left[\partial D_{z}\right] \neq 1$ in $\pi_{1}(\operatorname{Int} N(\partial B, \eta)-C)$.

We may assume that $F \mid\left(D^{2}-F^{-1}(C)\right)$ is PL. 
We now change $F$ by a PL approximation outside $D_{z}$ to obtain a map $F^{\prime}: D^{2} \rightarrow \operatorname{Int} B(y, \eta)$ such that

(1) $F^{\prime}\left(D^{2}-\right.$ Int $\left.D_{z}\right) \cap\{F(z)\}=\varnothing$;

(2) $F^{\prime} \mid\left(D^{2}-\{z\}\right)$ is PL;

(3) $F^{\prime}\left|D_{z}=F\right| D_{z}$.

(Note that very possible $F^{-1}(C)$ is not finite.)

Let $E_{k}$ be the disk on $\partial B$ such that $\partial E_{k}=\alpha_{k}$ and $\left[\partial B-E_{k} \cup \operatorname{Im} F^{\prime}\right] \neq 0$ in $\pi_{2}(\operatorname{Int} B(p, \theta)-\{p\})$. If necessary, we adjust $\partial B-\operatorname{Int} E_{k}$ slightly so that $\left(\partial B-\operatorname{Int} E_{k}\right) \cap\{F(z)\}=\varnothing$.

Now $\partial B-\operatorname{Int} E_{k} \cup \operatorname{Im} F^{\prime}-\{F(z)\}$ is the image of a proper PL map $g: \mathbf{R}^{2} \rightarrow$ Int $N(\partial B, \eta)-\{F(z)\}$ such that

(a) $\lim _{\|t\| \rightarrow \infty}\left\{g(t) \mid t \in \mathbf{R}^{2}\right\}=F(z)$,

(b) for some positive integer $m, g^{-1}(C) \cap\left\{t \in \mathbf{R}^{2} \mid\|t\|>m\right\}=\varnothing$.

Let $M$ be a locally regular neighborhood of $\operatorname{Im} g$ in $\operatorname{Int} N(\partial B, h)$ such that

(1) $\mathrm{Cl}(M)=M \cup\{F(z)\}$,

(2) for some $\xi>0$, Int $B(F(z), \xi) \cap(M \cap C)=\varnothing$.

Let $K=M \cap C$. Then $K$ is a compact subset of $M$. Let $H$ be the kernel of the inclusion-induced homomorphism $\pi_{1}(M-K) \rightarrow \pi_{1}(\operatorname{Int} N(\partial B, \eta)-C)$. Then $H$ is a normal subgroup of $\pi_{1}(M-K)$. For each positive integer $m$, let $T_{m}$ be the set $\left\{t \in \mathbf{R}^{2} \mid\|t\| \geq m\right\}$. Then, for $m$ large enough, $g^{-1}(C) \cap T_{m}=\varnothing$ and $\left[g \mid \partial T_{m}\right] \notin H$. For each positive integer $m$, let $S_{m}=\partial T_{m}$.

By the Plane Theorem of Brown and Feustel, there is a proper PL embedding in $h: \mathbf{R}^{2} \rightarrow M$ such that, for any large enough positive integer $m,\left[h \mid S_{m}\right] \notin H$. Since $h: \mathbf{R}^{2} \rightarrow M$ is a proper embedding, $\operatorname{Cl}(\operatorname{Im} h)=\operatorname{Im} h \cup\{F(z)\}$. Thus there is an embedding $h^{\prime}: S^{2} \rightarrow M \cup\{F(z)\}$, defined by

(a) $h^{\prime} \mid\left(\mathbf{R}^{2}-\{\infty\}\right)=h$,

(b) $h^{\prime}(\infty)=F(z)$.

For each positive integer $m$, let $D_{m}$ be the nonsingular disk $h^{\prime}\left(T_{m} \cup\{\infty\}\right)$. Let $x=F(z)=h^{\prime}(\infty)$. Choose $\varepsilon>0$ such that $B(x, \varepsilon) \subseteq \operatorname{Int} N(\partial B, \eta)$. For $m$ large enough, $D_{m} \subseteq$ Int $B(x, \varepsilon)$ and $D_{m} \cap C=\operatorname{Int} D_{m} \cap C=\{x\}$. Choose $m$ large enough and let $D=D_{m}$. Then $D$ is a nonsingular disk in $\operatorname{Int} B(x, \varepsilon)$ such that

(1) $D \cap C=\operatorname{Int} D \cap C=\{x\}$,

(2) $[\partial D] \neq 1$ in $\pi_{1}(\operatorname{Int} B(x, \varepsilon)-C)$.

This proves Theorem 1 .

Theorem 2. Let $C$ be a wild scrawny Cantor set in $\mathbf{R}^{3}$. Then $C$ is not definable by solid tori.

Proof. Suppose, on the contrary, that $C$ is definable by solid tori. By Theorem 1 , for some point $x$ of $C$ and some $\varepsilon>0$, there is a nonsingular disk $D$ in Int $B(x, \varepsilon)$ such that

(1) $D \cap C=\operatorname{Int} D \cap C=\{x\}$,

(2) $[\partial D] \neq 1$ in $\pi_{1}(\operatorname{Int} B(x, \varepsilon)-C)$.

It follows from condition (2) above that, for any $\eta>0$ small enough so that $\partial D \cap B(x, \eta)=\varnothing$ and for any PL 3-ball $B$ in Int $B(x, \eta)$ such that $x \in \operatorname{Int} B$, $\partial B \cap C \neq \varnothing$. Let $\eta>0$ be small enough that $\partial D \cap B(x, \eta)=\varnothing$. 
Since $C$ is definable by solid tori, there is a collection $\left\{V_{n} \mid n \in \mathbf{N}\right\}$ of solid tori such that, for each positive integer $n$,

(a) $\operatorname{diam} V_{n}<1 / n$,

(b) there is a PL 3-ball $B_{n}$ in Int $V_{n}$ such that $V_{n+1} \subseteq \operatorname{Int} B_{n}$;

(c) $\partial V_{n} \cap C=\varnothing$;

(d) $x \in \operatorname{Int} V_{n}$ and $\bigcap\left\{V_{n} \mid n \in N\right\}=\{x\}$;

(e) $V_{1} \subseteq \operatorname{Int} B(x, \eta)$.

Since $D \cap C=\{x\}$, for all $n \in \mathbf{N}, D \cap V_{n} \neq \varnothing$. For large enough $n$, $V_{n} \cap D \subseteq$ Int $D$. Let $n \in \mathbf{N}$ be large enough so that $V_{n} \cap D \subseteq$ Int $D$. We assume $D-\{x\}$ is PL and that, for each positive integer $k, D$ is in general position with respect to $\partial V_{k}$. Thus, for all $k \geq n, \partial V_{k} \cap D$ consists of a finite number of disjoint PL simple closed curves in Int $D$.

Let $k \geq n$; then $D \cap \partial V_{k}$ consists of finitely many PL simple closed curves in Int $D$. Since $x \in D \cap V_{k}$, there is an innermost component $\sigma$ of $D \cap \partial V_{k}$ such that

(1) $x \in$ Int $D_{\sigma}$, where $D_{\sigma}$ is the disk in Int $D$ such that $\partial D_{\sigma}=\sigma$;

(2) for each component $\gamma$ of Int $D_{\sigma} \cap \partial V k, x \notin D_{\gamma}$.

Since $\left[\partial D_{\sigma}\right]=[\partial D]$ in $\pi_{1}(\operatorname{Int} B(x, \varepsilon)-C),\left[\partial D_{\sigma}\right] \neq 1$ in $\pi_{1}(\operatorname{Int} B(x, \varepsilon)-C)$. We assume that $D_{\sigma}$ has been chosen so that the number of components of Int $D_{\sigma} \cap \partial V_{k}$ is minimal. Hence Int $D_{\sigma} \cap \partial V_{k}=\varnothing$.

For suppose that Int $D_{\sigma} \cap \partial V_{k} \neq \varnothing$. Let $\left\{\gamma_{j} \mid 1 \leq j \leq m\right\}$ be the set of components of Int $D_{\sigma} \cap \partial V_{k}$, and for each $1 \leq j \leq m$, let $D_{j}$ be the disk in Int $D_{\sigma}$ such that $\partial D_{j}=\gamma_{j}$. Since for each $1 \leq j \leq m\left[\gamma_{j}\right]=1$ in $\pi_{1}(\operatorname{Int} B(x, \varepsilon)-C)$ and since $\partial V_{k}$ is a torus and is incompressible in Int $B(x, \varepsilon)-C$, for each $1 \leq j \leq m$ there is a disk $E_{j}$ on $\partial V_{k}$ such that $\partial E_{j}=\gamma_{j}$. For some $1 \leq j \leq m, E_{j} \cap \bigcup\left\{\gamma_{j} \mid i \neq j\right\}=\varnothing$. Consider the disk $D_{\sigma}^{\prime}=D_{\sigma}-D_{j} \cup E_{j} . D_{\sigma}^{\prime}$ has all the virtues of $D_{\sigma}$, and $D_{\sigma}^{\prime} \cap \partial V_{k}$ has fewer components than $D_{\sigma} \cap \partial V_{k}$. This is impossible, by our choice of $D_{\sigma}$.

So $D_{\sigma} \cap \partial V_{k}=\partial D_{\sigma}=\sigma$.

We consider $D_{\sigma} \cap \partial V_{k+1}$. Let $\left\{\theta_{i} \mid 1 \leq i \leq t\right\}$ be the set of those components of $D_{\sigma} \cap \partial V_{k+1}$ such that, for each $1 \leq i \leq t,\left[\theta_{i}\right] \neq 1$ in $\pi_{1}(\operatorname{Int} B(x, \varepsilon)-C)$. We assume that $D_{\sigma}$ has been chosen so that the number of components of $D_{\sigma} \cap \partial V_{k+1}$ is minimal. Hence $D_{\sigma} \cap \partial V_{k+1}=\bigcup\left\{\theta_{i} \mid 1 \leq i \leq t\right\}$. Let the numbering be chosen so that, for all $1 \leq i \leq t, \theta_{i+1} \subseteq \operatorname{Int} D_{i}$, where $D_{i}$ is the disk in Int $D_{\sigma}$ such that $\partial D_{i}=\theta_{i}$.

For each $1 \leq i \leq t,\left[\theta_{i}\right] \neq 1$ in $\pi_{1}(\operatorname{Int} B(x, \varepsilon)-C)$. Hence, for each $1 \leq i \leq t,\left[\theta_{i}\right] \neq 1$ in $\pi_{1}\left(\partial V_{k+1}\right)$. Since $\partial V_{k+1}$ is a torus and since, for each $1 \leq i \neq j \leq t, \theta_{i} \cap \theta_{j}=\varnothing$, each $\theta_{i}$ is parallel to each $\theta_{j}$ on $\partial V_{k+1}$. That is, for each $1 \leq i \leq t,\left[\theta_{i}\right]=\left[\theta_{j}\right]$ in $\pi_{1}\left(\partial V_{k+1}\right)$.

Since $\theta_{1} \cup \sigma$ bounds an annulus in $V_{k}-$ Int $V_{k+1},\left[\theta_{1}\right]=[\sigma]$ in $\pi_{1}\left(V_{k}-\right.$ Int $\left.V_{k+1}\right)$. Since there is a PL 3-ball $B_{k}$ in Int $V_{k}$ such that $V_{k+1} \subseteq$ Int $B_{k},[\sigma]=1$ in $\pi_{1}\left(V_{k}-\right.$ Int $\left.V_{k+1}\right)$. Since $\left[\theta_{1}\right] \neq 1$ in $\pi_{1}\left(\partial V_{k+1}\right)$ and $\left[\theta_{1}\right]=1$ in $\pi_{1}\left(V_{k}-\right.$ Int $\left.V_{k+1}\right), \theta_{1}$ is a longitude of $V_{k+1}$.

The simple closed curve $\theta_{t}$ bounds the property embedded disk $D_{t}$ in $V_{k+1}$, and $\left[\theta_{t}\right] \neq 1$ in $\pi_{1}\left(\partial V_{k+1}\right)$. So $\theta_{t}$ is a meridian of $V_{k+1}$.

But we have already shown that $\left[\theta_{t}\right]=\left[\theta_{1}\right]$ in $\pi_{1}\left(\partial V_{k+1}\right)$. This is a contradiction.

So $C$ is not definable by solid tori. q.e.d. 


\section{FURTHER OBSERVATIONS AND AN EXAMPLE}

We begin with a definition suggested by Theorem 1 .

Definition. Let $C$ be a Cantor set in $\mathbf{R}^{3}$ and let $x \in C$. If, for some $\varepsilon>0$ there is a nonsingular disk $D$ in $\operatorname{Int} B(x, \varepsilon)$ such that $D \cap C=\operatorname{Int} D \cap C=\{x\}$ and $[\partial D] \neq 1$ in $\pi_{1}(\operatorname{Int} B(x, \varepsilon)-C)$, then we say that $C$ is sliceable at $x$.

Definition. Let $C$ be a Cantor set in $\mathbf{R}^{3}$ and let $X=\{x \in C \mid C$ is sliceable at $x$. Then $X$ is called the sliceable set of $C$.

Theorem D suggests the following

Definition. Let $C$ be a Cantor set in $\mathbf{R}^{3}$. Let $Y$ be the set $\{x \in C \mid$ for some $\varepsilon>0$, for any PL 3-ball $B$ in Int $B(x, \varepsilon), \partial B \cap C \neq \varnothing\}$. Then $Y$ is called the savage set of $C$.

For any Cantor set $C$, the savage set $Y$ is a dense subset of the wild set of $C$. Antoine's Eyeglasses is an example of a wild scrawny Cantor set in which $C=Y=X$. We have shown that, for any wild scrawny Cantor set $C$ in $\mathbf{R}^{3}$, each point of $Y$ is a limit point of $X$. Is it true that, for any wild scrawny Cantor set $C$ in $\mathbf{R}^{3}$ such that $C=Y, C$ is also equal to $X$ ? We give an example to show that it is false.

Let $C$ be the Cantor set in $\mathbf{R}^{3}$ with defining sequence $\left\{\mathscr{M}_{n}\right\}$ such that, for each positive integer $n$ and each $1 \leq k \leq m(n)$,

(1) $M_{n, k}$ is either a solid torus or a solid double torus;

(2) if $M_{n, k} \in \mathscr{M}_{n}$ is a solid torus, then $M_{n, k} \cap \bigcup \mathscr{M}_{n+1}$ is as shown in Figure 2;

(3) if $M_{n, k}$ is a solid double torus, then $M_{n, k} \cap \bigcup \mathscr{M}_{n+1}$ is as shown in Figure 1;

(4) $\mathscr{M}_{1}=\left\{M_{1,1}\right\}$ consists of one solid torus.

There is a point $p$ of $C$ such that the sequence of three-manifolds-withboundary $\left\{M_{n, k} \mid n \in \mathbf{N}, M_{n, k} \in \mathscr{M}_{n}\right.$, and $\left.p \in M_{n, k}\right\}$ is a sequence of solid tori. The proof of Theorem 1 shows that $C$ is not sliceable at $p$.

Thus it is not true, in general, that a wild scrawny Cantor set $C$ in $\mathbf{R}^{3}$ is sliceable at each point of its savage set.

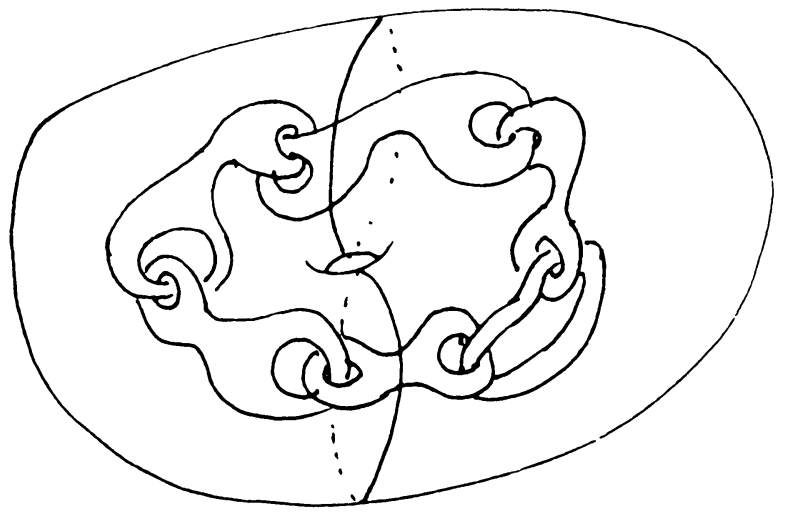

FIGURE 2 


\section{BIBLIOGRAPHY}

[A] L. Antoine, Sur l'homeomorphisme de deux figures et de leurs voisinages, J. Math. Pures Appl. 4 (1921), 221-325.

[Ba] A. Babich, Vorticist denizens of $\mathbf{R}^{3}$ are tame, $\mathrm{PhD}$ thesis, University of Texas at Austin, 1988.

[Bi] R. H. Bing, Tame Cantor sets in $E^{3}$, Pacific J. Math. 11 (1961), 435-446.

[Bl] W. A. Blankinship, Generalization of a construct of Antoine, Ann. of Math. (2) 53 (1951), 276-297.

[BF] E. M. Brown and C. D. Feustel, On properly embedding planes in arbitrary 3-manifolds, Proc. Amer. Math. Soc. 94 (1985), 173-178.

[D] R. J. Daverman, Decompositions of manifolds, Academic Press, Orlando, FL, 1986.

[H] T. Homma, On tame embedding on 0-dimensional compact sets in $E^{3}$, Yokohama Math. J. 7 (1959), 191-195.

[M] E. E. Moise, Geometric topology in dimensions 2 and 3, Springer-Verlag, New York, 1977.

4507 Red River Street, Austin, Texas 78751 